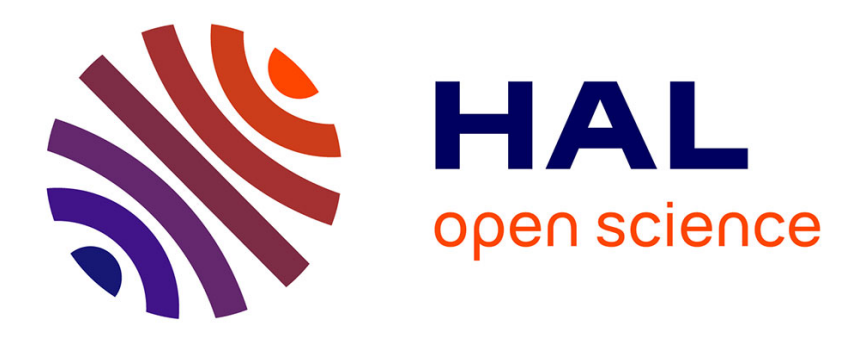

\title{
How to preserve the conflict as an alarm in the combination of belief functions?
}

\author{
Eric Lefevre, Zied Elouedi
}

\section{To cite this version:}

Eric Lefevre, Zied Elouedi. How to preserve the conflict as an alarm in the combination of belief functions?. Decision Support Systems, 2013, 56, pp.326-333. 10.1016/j.dss.2013.06.012 . hal-03354165

\section{HAL Id: hal-03354165 \\ https://hal.science/hal-03354165}

Submitted on 24 Sep 2021

HAL is a multi-disciplinary open access archive for the deposit and dissemination of scientific research documents, whether they are published or not. The documents may come from teaching and research institutions in France or abroad, or from public or private research centers.
L'archive ouverte pluridisciplinaire HAL, est destinée au dépôt et à la diffusion de documents scientifiques de niveau recherche, publiés ou non, émanant des établissements d'enseignement et de recherche français ou étrangers, des laboratoires publics ou privés. 


\title{
How to preserve the conflict as an alarm in the combination of belief functions?
}

\author{
Eric Lefèvre $^{\mathrm{a}}$, Zied Elouedi ${ }^{\mathrm{b}}$ \\ ${ }^{a}$ Univ. Lille Nord de France, UArtois, EA 3926 LGI2A, France \\ ${ }^{b}$ University of Tunis, Institut Supérieur de Gestion de Tunis, LARODEC, Tunisia
}

\begin{abstract}
In the belief function framework, a unique function is induced from the use of a combination rule so allowing to synthesize all the knowledge of the initial belief functions. When information sources are reliable and independent, the conjunctive rule of combination, proposed by Smets, may be used. This rule is equivalent to the Dempster rule without the normalization process. The conjunctive combination provides interesting properties, as the commutativity and the associativity. However, it is characterized by having the empty set, called also the conflict, as an absorbing element. So, when we apply a significant number of conjunctive combinations, the mass assigned to the conflict tends to 1 which makes impossible returning the distinction between the problem arisen during the fusion and the effect due to the absorption power of the empty set.

The objective of this paper is then to define a formalism preserving the initial role of the conflict as an alarm signal announcing that there is a kind of disagreement between sources. More exactly, that allows to preserve some conflict, after the fusion by keeping only the part of conflict reflecting the opposition between the belief functions. This approach is based on dissimilarity measures and on a normalization process between belief functions. Our proposed formalism is tested and compared with the conjunctive rule of combination on synthetic belief functions.
\end{abstract}

Keywords: Belief function theory, Data fusion, Alarm of singular source, Conflict management.

Email addresses: eric.lefevre@univ-artois.fr (Eric Lefèvre), zied.elouedi@gmx.fr (Zied Elouedi) 


\section{Introduction}

Since many years, the belief function theory $[8,43]$ has known an increasing interest from scientific community since it allows to deal with imperfect data (imprecise and uncertain) and to combine them using a combination rule. One of the classical combination rules is the conjunctive rule. This latter, introduced by Smets $[44,48]$, is equivalent to the Dempster rule of combination $[8,43]$ without the normalization process. Properties and also hypotheses that sources should satisfy before being combined by this rule are well established.

This rule has an orthogonal behavior which is very precious because it permits a fast and clear convergence towards a solution, but in return, the empty set is an absorbing element. Smets supports that the existence of this mass on the empty set, called also conflict, can play a role of alarm. So, contrary to Dempster's rule where the conflict is reallocated proportionally to the other masses of the focal elements, this conflict must not be redistributed since it may be at the origin of important information concerning the progress of the fusion process and show the disagreement between sources. In fact, if the conflict is small, it means that the joint bba fits with the opinions given by the sources to fuse and consequently try to reinforce them, whereas when the conflict is high, it means that the induced bba is largely in contradiction with the previous opinions. Nevertheless, due to its absorbing conjunctive effect, a series of combinations aims at getting the empty set equal to 1 , making impossible the distinction between a real problem between sources to fuse and an effect caused by the absorbing of the empty set.

In addition to the conflict definition of Smets, other works have been dealt with the conflict definition namely Liu [30] proposes a quantitative measure taking into account the mass on the empty set induced from the combination of two or more bbas and the distance between betting commitments of these same bbas after applying the pignistic transformation. However, this mass on the empty set remains not sufficient to exactly express the conflict. On the other hand, in [38], Osswald and Martin present another interpretation of the conflict by defining the auto-conflict as the amount of intrinsic conflict of a belief function, in other words it is the conflict generated by such a function relative to one information source.

Besides in [11], Destercke and Burger present some properties that a mea- 
sure of extrinsic conflict should satisfy. They defined conflict as the inconsistency arising from a conjunctive combination, and based on properties, they also proposed conflict measurements making no a priori assumptions regarding the dependence between sources.

Thus, two types of conflict can be defined:

- The conflict which allows the estimation of the confusion rate of a source and which will be called intrinsic conflict [5, 20, 38, 42],

- The conflict which evaluates the discordance between two bodies of evidence and will be labeled extrinsic conflict [23, 39, 49].

In this paper, we characterize the opposition between belief functions by means of a measure of dissimilarity. This measure is then used in our proposed approach named Combination With Adapted Conflict (CWAC) providing an adaptive weighting between Dempster's rule and conjunctive rule, allowing to keep the initial meaning of the conflict obtained during the combination and so to restore its initial role of alarm. Thus, it permits to the conflict to take back its initial sense by only mentioning that there is a problem somewhere and reducing its absorbing power. Our proposal is not a conflict measure but a combination rule preserving the main role of a conflict as a signal making aware of this opposition between sources. A preliminary work of this approach has been proposed in [29].

This paper is organized as follows. Section 2 presents the basics of the belief function theory. Combination rules, proposed in the belief function framework, are detailed in Section 3. The definition and properties of our CWAC rule are exposed in section 4 . Section 5 brings to light our proposed approach by comparing its behavior with that of the conjunctive combination in the case of synthetic data. Section 6 concludes our study and presents some future works.

\section{Belief function theory: background}

The belief function theory is considered as a useful theory for representing and managing uncertain knowledge. In this Section, we shall briefly recall some basics of this theory. More details can be found in [43, 44, 48]. 


\subsection{Representing information}

Let $\Omega$ be a finite non empty set including all the elementary events related to a given problem. These events are assumed to be exhaustive and mutually exclusive. Such set $\Omega$ is named frame of discernment.

The impact of a piece of evidence on the different subsets of the frame of discernment $\Omega$ is represented by the so-called basic belief assignment (bba), called initially by Shafer [43] basic probability assignment.

The bba $m$ is a function $m: 2^{\Omega} \rightarrow[0,1]$ that satisfies:

$$
\sum_{A \subseteq \Omega} m(A)=1
$$

The basic belief mass $m(A)$, expresses the part of belief exactly committed to the event $A$ of $\Omega$ given a piece of evidence. Due to the lack of information, this quantity cannot be apportioned to any strict subset of $A$.

Shafer [43] has initially proposed a normality condition expressed by:

$$
m(\emptyset)=0
$$

Such bba is called a normalized basic belief assignment.

Smets $[44,45]$ relaxes this condition by considering $m(\emptyset)$ as the amount of conflict between the pieces of evidence or as the part of belief given to the fact that none of the hypotheses in $\Omega$ is true. All the subsets $A$ of $\Omega$ such that $m(A)$ is strictly positive, are called the focal elements of $m$.

Associated with $m$ is the belief function, denoted bel, corresponding to a specific bba $m$, assigns to every subset $A$ of $\Omega$ the sum of masses of belief committed to every subset of $A$ by $m$ [43]. This belief function, bel, represents the total belief that one commits to $A$ without being also committed to $\bar{A}$. The belief function bel : $2^{\Omega} \rightarrow[0,1]$ is defined so that:

$$
\begin{gathered}
\operatorname{bel}(A)=\sum_{\emptyset \neq B \subseteq A} m(B), \forall A \subseteq \Omega \\
\operatorname{bel}(\emptyset)=0
\end{gathered}
$$

The plausibility function $p l: 2^{\Omega} \rightarrow[0,1]$ quantifies the maximum amount of belief that could be given to a subset $A$ of $\Omega$. It is equal to the sum of the masses given to subsets $B$ compatible with $A$ :

$$
\begin{gathered}
p l(A)=\sum_{A \cap B \neq \emptyset} m(B), \forall A \subseteq \Omega \\
p l(\emptyset)=0
\end{gathered}
$$




\subsection{Special belief functions}

In this subsection, we propose some belief functions used to express particular situations related generally to uncertainty. A vacuous bba is defined as follows [43]:

$$
m(\Omega)=1 \text { and } m(A)=0 \quad \forall A \neq \Omega
$$

Such function quantifies the state of total ignorance by having only $\Omega$ as a focal element.

A categorical bba is a normalized bba defined as follows:

$$
m(A)=1 \quad \forall A \subset \Omega \quad \text { and } \quad m(B)=0 \quad \forall B \subseteq \Omega, B \neq A
$$

This function has a unique focal element different from the frame of discernment $\Omega$.

A certain bba is a particular categorical bba such that its focal element is a singleton. A certain bba is defined as follows:

$$
m(A)=1 \text { and } m(B)=0 \quad \forall B \neq A \quad \text { and } \quad B \subseteq \Omega \quad \text { and } \quad|A|=1
$$

where $A$ is a singleton event of $\Omega$. This function represents a state of total certainty on the focal element.

A simple support function (ssf) if it has at most one focal element different from the frame of discernment $\Omega$. A simple support function is defined as follows [46]:

$$
m(X)=\left\{\begin{array}{ll}
w & \text { if } X=\Omega \\
1-w & \text { if } X=A \quad \forall A \subseteq \Omega \\
0 & \text { otherwise }
\end{array} \quad \forall\right.
$$

where $A$ is the focal element and $w \in[0,1]$. It presents a belief function induced by a piece of evidence supporting $A$ (with $1-w$ ) and leaving the remaining beliefs for $\Omega$. This bba can also be noted $A^{w}$.

A Bayesian bba is a particular case of belief functions where all the focal elements are singletons. The corresponding bba is defined as follows:

$$
m(A)>0 \text { only when }|A|=1
$$

In this case, $b e l=p l$ and they are considered as a probability distribution.

A consonant bba is a bba when all its focal elements $\left(A_{1}, A_{2}, \ldots, A_{n}\right)$ are nested, that is $A_{1} \subseteq A_{2} \subseteq \ldots \subseteq A_{n}$. It is a special case of possibilities.

A dogmatic belief function is defined such that $m(\Omega)=0$. Inversely a non-dogmatic belief function is defined such that $m(\Omega)>0[46]$. 


\subsection{The discounting operation}

Handling evidence given by experts requires to take into account the level of expertise of each information source. Indeed, reliability differs from one expert to another and a discounting method is imperative to update experts' beliefs based on weighting most heavily the opinions of the best experts and conversely for the less reliable ones.

Let $\alpha \in[0,1]$, be the discounting rate, and consequently $1-\alpha$ is regarded as a degree of confidence on the expert. Updating the expert's opinions leads to:

$$
\left\{\begin{array}{l}
m^{\alpha}(A)=(1-\alpha) m(A), \quad \forall A \subset \Omega \\
m^{\alpha}(\Omega)=(1-\alpha) m(\Omega)+\alpha
\end{array}\right.
$$

The larger $\alpha$, the closer $m^{\alpha}$ is from the vacuous belief function. So, when $\alpha=1$, it means that the expert is not reliable at all, and his opinions should be totally ignored. On the other hand, when $\alpha=0$, it means that the expert is totally reliable.

\subsection{Pignistic transformation}

To make a decision, Smets proposes to transform beliefs to a probability measure, denoted BetP [48]. The link between these two measures is achieved by the pignistic transformation such that:

$$
\operatorname{Bet} P(A)=\sum_{B \subseteq \Omega} \frac{|A \cap B|}{|B|} \frac{m(B)}{1-m(\emptyset)}, \forall A \in \Omega .
$$

$B e t P$ can be extended as a function on $2^{\Omega}$ as:

$$
\operatorname{Bet} P(A)=\sum_{\omega \in A} \operatorname{Bet} P(\omega) \quad \forall A \subseteq \Omega \text { and } \omega \in \Omega .
$$

\subsection{Distance between bbas}

Several researchers have proposed distances under a belief function framework. Some of them are based on the pignistic transformation $[1,16,49,55]$, where an unavoidable step is the pignistic transformation of the bbas. On the other hand, some distances based directly on bbas, are also proposed [17, 23] defined on the power set of the frame of discernment.

In this paper, we will focus on the Jousselme distance [23] considered as one of the most known distances. 
Let $m_{1}$ and $m_{2}$ two bbas, the Jousselme distance [23] is defined as follows:

$$
d_{J}\left(m_{1}, m_{2}\right)=\sqrt{\frac{1}{2}\left(m_{1}-m_{2}\right)^{t} \mathcal{D}\left(m_{1}-m_{2}\right)}
$$

where $\mathcal{D}$ is the Jaccard index defined by:

$$
\mathcal{D}(A, B)=\left\{\begin{array}{cl}
0 & \text { if } A=B=\emptyset \\
\frac{|A \cap B|}{|A \cup B|} & \forall A, B \in 2^{\Omega} .
\end{array}\right.
$$

\section{Combination of pieces of evidence}

In the case of imperfect data (uncertain, imprecise and incomplete), data fusion becomes an interesting solution allowing to obtain more relevant information. The belief function theory offers appropriate tools for ensuring fusion. The objective of the combination is then to synthesize the information issued of a set of belief functions in a unique function. Based on the fusion of bbas induced from several sources, the conflict between these sources may be arisen. The conflict is defined as a measure of a disagreement between the sources. In the belief function framework, several combination approaches were developed. They can be classified in two categories according whether sources are reliable or not.

\subsection{Reliable sources}

When information sources, inducing belief functions, are considered as reliable, the used operators are based on the conjunctive combination. Hence, the induced result of the conjunctive combinations of two bbas $m_{1}$ and $m_{2}$, is noted $m_{\ominus}$ and is defined by [48]:

$$
m_{\circledast}(A)=\sum_{B \cap C=A} m_{1}(B) m_{2}(C) \quad \forall A, B, C \subseteq \Omega .
$$

This rule considers $m_{\ominus}(\emptyset)$ as the discord between sources implied in the fusion process and called it conflict.

Another kind of conjunctive combination is Dempster's rule [8], based on the orthogonal sum, and is considered as the standard fusion rule used in the case of reliable sources. The induced bba is noted $m_{\oplus}$ and is defined by:

$$
m_{\oplus}(A)=\frac{1}{1-m_{\ominus}(\emptyset)} m_{\circledast}(A) \quad \forall A \neq \emptyset \quad \text { and } \quad m_{\oplus}(\emptyset)=0
$$


where $\frac{1}{1-m @(\emptyset)}$ is the normalization factor making $m_{\oplus}(\emptyset)=0$.

These two combination rules namely the conjunctive and the Dempster rules are commutative and associative but not idempotent. Therefore, they cannot be used when the sources are not independent ${ }^{1}$.

In addition, Denœux [10] has developed the cautious conjunctive rule having also a conjunctival behavior. This rule is idempotent, and can consequently be applied when information sources are dependent. It is defined by the following relation:

$$
m_{1} \oslash m_{2}=\bigcap_{A \subset \Omega} A^{\omega_{1}(A) \wedge \omega_{2}(A)}
$$

where $\wedge$ is the minimum operator and $\omega_{i}$ are the weights obtained from the decomposition based on the conjunctive combination of $m_{i}$ in simple support functions such as:

$$
m_{i}=\bigcap_{A \subset \Omega} A^{\omega_{i}(A)}
$$

It is useful to note that these various combinations can also be applied even in the case of unreliable information sources. In such a case, a discounting is then necessary to be applied before the combination. This approach was used in several works $[36,50]$. The difficulty remains then in the assessment of the degrees of reliability. There are several strategies to estimate this reliability. One of them is based on the calculation of a measure of dissimilarity between the belief functions to be combined. This measure is used as a degree of discounting. Hence, contradictory belief functions are discounted before fusion phase. This approach was studied by several authors $[26,31,32,33,41,53]$. Another technique consists in using further information on the belief functions to define the discounting degree coefficients $[15,16]$.

\subsection{Unreliable sources}

If one source in the fusion process is not reliable and there is no possible adjustment then combinations based exclusively on the conjunctive rule cannot be used. As a first rule dealing with such a case, is the disjunctive

\footnotetext{
${ }^{1}$ The notion of independence is relatively delicate to define. We consider here the idea of cognitive independence [45].
} 
rule of combination proposed by Dubois and Prade [12]. Then, in the case of two bbas $m_{1}$ and $m_{2}$, this rule is defined by:

$$
m_{(}(A)=\sum_{B \cup C=A} m_{1}(B) m_{2}(C) \quad \forall A, B, C \subseteq \Omega .
$$

This kind combination is the dual of the conjunctive combination. It is discussed within the Generalized Bayes Theorem by Smets [45]. As the conjunctive one, this rule is also associative and commutative but not idempotent. In the case where sources are dependent, Denœux [10] suggests using the bold disjunctive rule. For combining two bbas $m_{1}$ and $m_{2}$, this rule is defined in the following way:

$$
m_{1} \oslash m_{2}=\underset{A \neq \emptyset}{\bigoplus} A_{\nu_{1}(A) \wedge \nu_{2}(A)}
$$

where $\nu_{1}$ and $\nu_{2}$ represent the disjunctive decomposition ${ }^{2}$ of $m_{1}$ and $m_{2}$ based on simple generalized masses such that:

$$
m_{i}=\underset{A \neq \emptyset}{(\cup)} A_{\nu_{i}(A)} .
$$

Besides, rules having intermediate behavior between the conjunctive and disjunctive rules of combination were developed these last years [7, 13, 14, 19]. Among these rules, we mention the one introduced by Florea [19] which allows to define a family of rules having an intermediate behavior between conjunctive and disjunctive operators according to two functions $\gamma_{1}$ and $\gamma_{2}$ dependent on the conflict $m_{\ominus}(\emptyset)$. This rule is defined in the following way:

$$
m_{F}(A)=\gamma_{1}\left(m_{\oplus}(\emptyset)\right) m_{\circledast}(A)+\gamma_{2}\left(m_{\circledast}(\emptyset)\right) m_{(}(A) \quad \forall A \neq \emptyset
$$

Several definitions for the functions $\gamma_{1}$ and $\gamma_{2}$ were proposed. However, the authors recommend the use of logarithmic forms which better correspond to the non-symmetric distribution of the conflict. This rule is called robust rule of combination. For other combination rules, it is a question to redistribute the partial conflict $[4,18,35,52]$. Finally, we can also note the other rules, less used, which allow too to combine bbas $[21,22,37,51,54]$. The objective of all these rules is to redistribute the conflict induced during the combination.

\footnotetext{
${ }^{2}$ The function $\nu_{i}$ is the disjunctive counterpart of function $\omega$, see $[9,10]$ for a detailed definition of function $\nu$.
} 


\subsection{Discussion}

Some tricky situations may often happen, when it is necessary to combine a set of sources where some of them are contradictory. The term of singular sources can be defined for sources that supply different information of what the other sources propose [27]. So, being singular or not depends on the fact to be in agreement with the majority. This discord with the majority can result $[28,47]$ :

- That the source at the origin of the discord is not reliable. For example, a failing sensor or working outside his range of functioning can disagree with the other information sources. In that case, the information induced from this source is not relevant.

- Or what this source possesses as information not perceived by the others. For example in the case of the detection of target, several sensors cannot see a target hidden by an obstacle while a source positioned in a different way sees perfectly this target. This source is then very instructive.

So, for a given application, the selection of a combination operator, among those proposed, is relatively sensitive to handle. In fact, in most of the situations, it is impossible to know if some sources are (or will become) reliable or not reliable. The use of a strategy of combination of reliable sources (for example conjunctive rule) containing one or several non-informative singular sources is so going to end in a process of less robust fusion. In the same manner, the use of a combination of unreliable sources with informative singular sources means, generally, redistributing some conflict. Redistribution which may be likened to a loss of information.

To identify the situation and thus select the appropriate combination, recent works $[6,27,30,33,40,41]$ introduced measures of conflict, other than the value of $m(\emptyset)$, allowing to quantify the opposition between belief functions. However, these measures are decoupled by the combination belief functions. So, these solutions produce a set of measures. Each of these measures reflecting the opposition between a source and the other sources involved in the process of fusion. This set of measures remains still difficult to interpret and thus the identification of the situation (reliable or unreliable sources) is tricky. In the following section, a formalism, which allows to protect the part of opposition between belief functions during the combination, 
is presented. This formalism supplies an alarm to the decision maker, concerning his process of fusion, allowing him to choose adequate measures as for example to strengthen the weight of the singular source if it is informative or to treat it as an erroneous source in the opposite case. This approach allows to obtain the result of the combination and a measure of the quality of the fusion process.

\section{Combination with adapted conflict (CWAC)}

In this paper, belief functions are considered as outcomes of independent information sources. In this frame, the conflict $m(\emptyset)$ obtained during the combination of belief functions allows to draw the attention on a possible problem like bad modelling, to an unreliable source now and more generally the presence of a singular source. Most of the combinations proposed in the literature (see Section 3) try to redistribute this conflict and not to use it as indicator. Only the conjunctive combination allows to preserve the mass on the empty set.

However, when the conjunctive combination is applied on a large number of belief functions, the conflict can take important proportions without reflecting a real problem. In such a case, this value does not represent a real opposition. This phenomenon is due to the absorbing character of the empty set.

Based on this analysis, we intend to develop a method allowing to transform the mass on the empty set as a real indicator of problems even if the number of sources to be combined is important. Our approach is named Combination With Adapted Conflict (CWAC).

Considering that there is a problem when sources produce strongly different belief functions, the conflict must be kept during the fusion. On the contrary, in the case of the combination of information sources for which the distributions of masses are equivalent, the conflict does not have to exist. The spirit of CWAC is to keep the conflict exclusively reflecting the disagreement between sources without taking into account the part that could be called auto-conflict is defined for only one source [34, 38]. To define CWAC rule, a measure allowing to determine the resemblance between bbas is necessary.

So, one of the interests of our CWAC rule is to detect the unreliable source(s). In fact, an information source is not usually reliable and its reliability may change from time to time. Two solutions are then possible. The first one consists in checking before fusing if each source is reliable or 
not. It is then necessary to compute the reliability of each source at each time and then fuse them (if sources are identified enough as reliable). The second solution has only one phase, using CWAC for combination, so get at the meantime, the fusion result and the disagreement measure (so potentially the unreliability of a source). According to the value of this measure, the expert may accept or not the results of the fusion. If he does not accept it, he should then look for the unreliable source(s) (like the first solution). Hence, our proposed solution needs less time than the first one.

So, through CWAC, we can discover this through the mass on the empty set, whereas as mentioned using the conjunctive rule, it is not so obvious due to the absorbing behavior of the empty set and also especially when the number of the sources is important.

\subsection{With two belief functions}

At first, only the case of two bbas $m_{1}$ and $m_{2}$ is considered. The dissimilarity notion may be obtained from a measure of distance. This distance may be calculated by the method proposed in section 2.5 and is noted $d\left(m_{1}, m_{2}\right)^{3}$. The borders of the function $d$ are:

- $d\left(m_{1}, m_{2}\right)=0: m_{1}$ and $m_{2}$ are similar (and consequently in agreement) and their combination should not generate a conflict. In this case, the conflict must be redistributed in the same manner as the combination rule of Dempster.

- $d\left(m_{1}, m_{2}\right)=1: m_{1}$ and $m_{2}$ are antinomic (i.e. $m_{1}\left(\left\{\omega_{j}\right\}\right)=1$ and $m_{2}\left(\left\{\omega_{i}\right\}\right)=1$ with $\omega_{i} \neq \omega_{j}, \omega_{i} \in \Omega$ and $\left.\omega_{j} \in \Omega\right)$. Their combination produces a conflictual mass expressing their opposition. This value must be kept in the same way as with the conjunctive combination.

CWAC combination is defined by an adaptive weighting between the conjunctive and Dempster's rules. This adaptive weighting allows to obtain a behavior similar to that of the conjunctive rule when belief functions are

\footnotetext{
${ }^{3}$ However, the other measures of dissimilarity could be used $[2,3]$. Details of the measures of dissimilarity are given in $[24,25]$. Besides, the use of measures of conflict, as that proposed in [27], can be considered. The objective of this paper is not to compare these approaches but simply to use one of these measures to supply to the decision maker a unique belief function, induced from the combination, containing a part of belief on the revealing empty set of the existing opposition.
} 
antinomic and equivalent to that of the Dempster rule when belief functions are similar. Between these two extremes, a gradual passage may be considered. The combination rule which we propose noted $\Theta$ can be written then in the following way:

$$
m_{\Theta}(A)=\gamma_{1} m_{\oplus}(A)+\gamma_{2} m_{\oplus}(A) \quad \forall A \subseteq \Omega
$$

with:

$$
\begin{aligned}
m_{\oplus}(A) & =\left(m_{1} \oplus m_{2}\right)(A) & \forall A \subseteq \Omega \\
m_{\circledast}(A) & =\left(m_{1} @ m_{2}\right)(A) & \forall A \subseteq \Omega
\end{aligned}
$$

where $\gamma_{1}$ and $\gamma_{2}$ are dependent functions of distance $d\left(m_{1}, m_{2}\right)$. These functions should satisfy the following constraints:

$$
\begin{aligned}
& \gamma_{1}=f_{1}\left(d\left(m_{1}, m_{2}\right)\right) \quad \text { with } \quad f_{1}(0)=0 \quad \text { and } \quad f_{1}(1)=1 \\
& \gamma_{2}=f_{2}\left(d\left(m_{1}, m_{2}\right)\right) \quad \text { with } \quad f_{2}(0)=1 \quad \text { and } \quad f_{2}(1)=0
\end{aligned}
$$

with $\gamma_{1}+\gamma_{2}=1$. However other functions are possible, we can choose at first, linear functions such that:

$$
\begin{array}{r}
\gamma_{1}=d\left(m_{1}, m_{2}\right) \\
\gamma_{2}=1-d\left(m_{1}, m_{2}\right) .
\end{array}
$$

Hence, our rule can be written $\forall A \subseteq \Omega$ and $m_{\curvearrowleft}(\emptyset) \neq 1$ :

$$
m_{\Theta}(A)=m_{1} \Theta m_{2}(A)=d\left(m_{1}, m_{2}\right) m_{\oplus}(A)+\left(1-d\left(m_{1}, m_{2}\right)\right) m_{\oplus}(A) .
$$

When $m_{\oplus}(\emptyset)=1$, then we have $m_{\Theta}(\emptyset)=1$.

\subsection{General case}

The generalization of this approach arises when more than two bbas are to be merged. Indeed, in a classic way the measures of dissimilarity are defined only between two bbas functions. Let $N$ bbas noted $m_{1}, \ldots, m_{i} \ldots, m_{N}$ which should be fused. The measure of dissimilarity between these functions, which it is necessary to use in the case of the proposed approach, may be a synthesis of the distances between bbas. The objective of the proposed approach is to offer to the decision maker, after the fusion, a measure of conflict which allows to distinguish the presence of a singular source among bbas. It seems 
then natural to make this synthesis by using, for example, the maximal value of the set of distances ${ }^{4}$. In that case, the value of $D$ may be defined by:

$$
D=\max _{i, j}\left[d\left(m_{i}, m_{j}\right)\right]
$$

with $i \in[1, N]$ and $j \in[1, N]$. Our rule can be generalized as the following manner $\forall A \subseteq \Omega$ and $m_{\curvearrowleft}(\emptyset) \neq 1$ :

$$
m_{\Theta}(A)=\left(\Theta_{i} m_{i}\right)(A)=D m_{\oplus}(A)+(1-D) m_{\oplus}(A)
$$

and

$$
m_{\oplus}(\emptyset)=1 \quad \text { when } \quad m_{\curvearrowleft}(\emptyset)=1
$$

with:

$$
m_{\oplus}(A)=\left(\bigoplus_{i} m_{i}\right)(A) \quad \text { and } \quad m_{\oplus}(A)=\left(\underset{i}{\oplus} m_{i}\right)(A) \quad \forall i \in[1, N] .
$$

\subsection{Properties}

Here are some properties characterizing our CWAC rule:

- Commutativity: CWAC is commutative, for all $m_{1}, m_{2}$ :

$$
m_{1} \Theta m_{2}=m_{2} \Theta m_{1} .
$$

- Associativity: CWAC is not associative, for all $m_{1}, m_{2}, m_{3}$ :

$$
m_{1} \Theta\left(m_{2} \Theta m_{3}\right) \neq\left(m_{1} \Theta m_{2}\right) \Theta m_{3} .
$$

- Neutral element: The neutral element of the CWAC is $\Omega$. Let $m_{0}$ be the vacuous bba. So for all $m$ :

$$
m \Theta m_{0}=m .
$$

- Absorbing element: The absorbing element of the CWAC is $\emptyset$. Let $m_{e}$ be a bba having $\emptyset$ has the unique focal element $(m(\emptyset)=1)$. So, for all $m$ :

$$
m \oplus m_{e}=m_{e} .
$$

- Idempotent: CWAC is not idempotent, for all $m$ :

$$
m \oplus m \neq m .
$$

\footnotetext{
${ }^{4}$ According to the wished behavior, the decision maker can use the other approaches (such as the average for example) to synthesize the measures of dissimilarity.
} 


\section{Experimental results}

In this Section, the proposed combination is compared to the conjunctive combination on synthetic data. The first experimentation more emphasizes on the study of the behavior of the different operators in the case of a reduced number of belief functions. Then, the second and the third experimentations allow to study the behavior of the operators according to the number of bbas and to the similarity between them.

\subsection{Example $n^{\circ} 1$}

For this first experimentation, we consider a frame of discernment $\Omega=$ $\left\{\omega_{1}, \omega_{2}, \omega_{3}\right\}$. At first, we consider 5 bbas defined in Table 1 . We notice that these bbas are relatively similar. In this Table, we find the results of the combination of these 5 sources with the conjunctive operator $\left(m_{\oplus}^{1, \ldots, 5}\right)$ and our CWAC operator $\left({ }^{J} m_{\Theta}^{1, \ldots, 5}\right.$ with Jousselme's distance). The conflict induced by the conjunctive combination is relatively important which is not the case with our approach (0.9317 against 0.1118 if we use the Jousselme distance).

Now, let us consider the case, where a 6th source is added. This source is singular with regard to 5 first ones. Results of the combination of these 6 bbas are also presented in Table 1 . These results allow to deduce that there is an important increase of the value of the conflict in the case of our proposed approach. This value is, according to the used distance, from 5 to 6 times more important than during the first combination. On the other hand, in the conjunctive combination case, the increase is only $6.4 \%$ (that is only 1.06 more important). So, the detection of the presence of a singular source in the fusion process is more easier with our CWAC operator. These conclusions are consolidated by the experimentations presented in the following sections.

\subsection{Example $n^{\circ} 2$}

For this experimentation, we are going to study the evolution of the conflict, according to the maximal distance, in a set of $N$ belief functions. The focal elements as well as degrees of beliefs are randomly generated according to a normal law. The focal elements are altogether selected in $2^{\Omega}$ with the cardinal of $\Omega$ equal to 3 . Results presented on Figure 1 are the average of 100 experiences. In that case, the behavior of our proposal is almost linear according to the maximal distance. This conclusion is usually true for any chosen distance and also for any number of belief functions involved in 


\begin{tabular}{|c|c|c|c|c|c|c|c|c|}
\hline bba & $\emptyset$ & $\left\{\omega_{1}\right\}$ & $\left\{\omega_{2}\right\}$ & $\left\{\omega_{1}, \omega_{2}\right\}$ & $\left\{\omega_{3}\right\}$ & $\left\{\omega_{1}, \omega_{3}\right\}$ & $\left\{\omega_{2}, \omega_{3}\right\}$ & $\Omega$ \\
\hline$m_{1}$ & 0 & 0.55 & 0 & 0 & 0.40 & 0 & 0.05 & 0 \\
\hline$m_{2}$ & 0 & 0.50 & 0 & 0 & 0.30 & 0 & 0.2 & 0 \\
\hline$m_{3}$ & 0 & 0.60 & 0 & 0 & 0.25 & 0. & 0.15 & 0 \\
\hline$m_{4}$ & 0 & 0.52 & 0 & 0 & 0.25 & 0 & 0.23 & 0 \\
\hline$m_{5}$ & 0 & 0.59 & 0 & 0 & 0.22 & 0 & 0.19 & 0 \\
\hline$m_{(\mathfrak{0}}^{1} \ldots, 5$ & 0.9317 & 0.0506 & 0 & 0 & 0.0176 & 0 & 0.0001 & 0 \\
\hline${ }^{J} m_{\Theta}^{1} \ldots, 5$ & 0.1118 & 0.6580 & 0 & 0 & 0.2294 & 0 & 0.0009 & 0 \\
\hline$m_{6}$ & 0 & 0 & 0.5 & 0 & 0.30 & 0 & 0.2 & 0 \\
\hline$m_{\overparen{G}}^{1} \ldots ., 6$ & 0.9911 & 0.0001 & 0 & 0 & 0.0088 & 0 & 0 & 0 \\
\hline${ }^{J} m_{\Theta}^{1} \ldots, 6$ & 0.5628 & 0.0016 & 0 & 0 & 0.4349 & 0 & 0.0006 & 0 \\
\hline
\end{tabular}

Table 1: Behavior of the different combinations

the fusion process. In case with more than 10 belief functions and when the maximum distance is greater than 0.5 , the conjunctive combination produces already a conflictual mass close to 1 , this is not the case for our proposal. In this situation, the proposed approach yet allows us to distinguish the opposition between the belief functions.

We can also note that when the maximal distance is low, the conflict is almost null. On the contrary, and in the same case, the use of the conjunctive combination induces a significant conflict (superior or equal to 0.5 according to the number of the belief functions combined) while bbas are similar. Furthermore, the convergence towards a maximal value of conflict is fast as the number of bbas is important.

While both approaches have the same behavior, the dynamics obtained by CWAC function is more important. Indeed, the combination CWAC, the conflicting mass ranges from 0 (no conflict) to 0.8 (conflict situation). In this case, the dynamics is then 0.8 . With the conjunctive combination in the best case, this value is only 0.5 . With a variation range greater, it is more easily to define the threshold at which the system can be questioned (unreliable source or singular source). Finally, for a given combination rule, the results vary according to the number of sources $(N=10,20$ or 30). However, these variations are more important in the case of conjunctive combination and remain very limited with CWAC.

\subsection{Example $n^{\circ} 3$}

In this experimentation, two sets of belief functions $S_{1}$ and $S_{2}$ are considered. Within every set, bbas are identical. On the other hand, between 


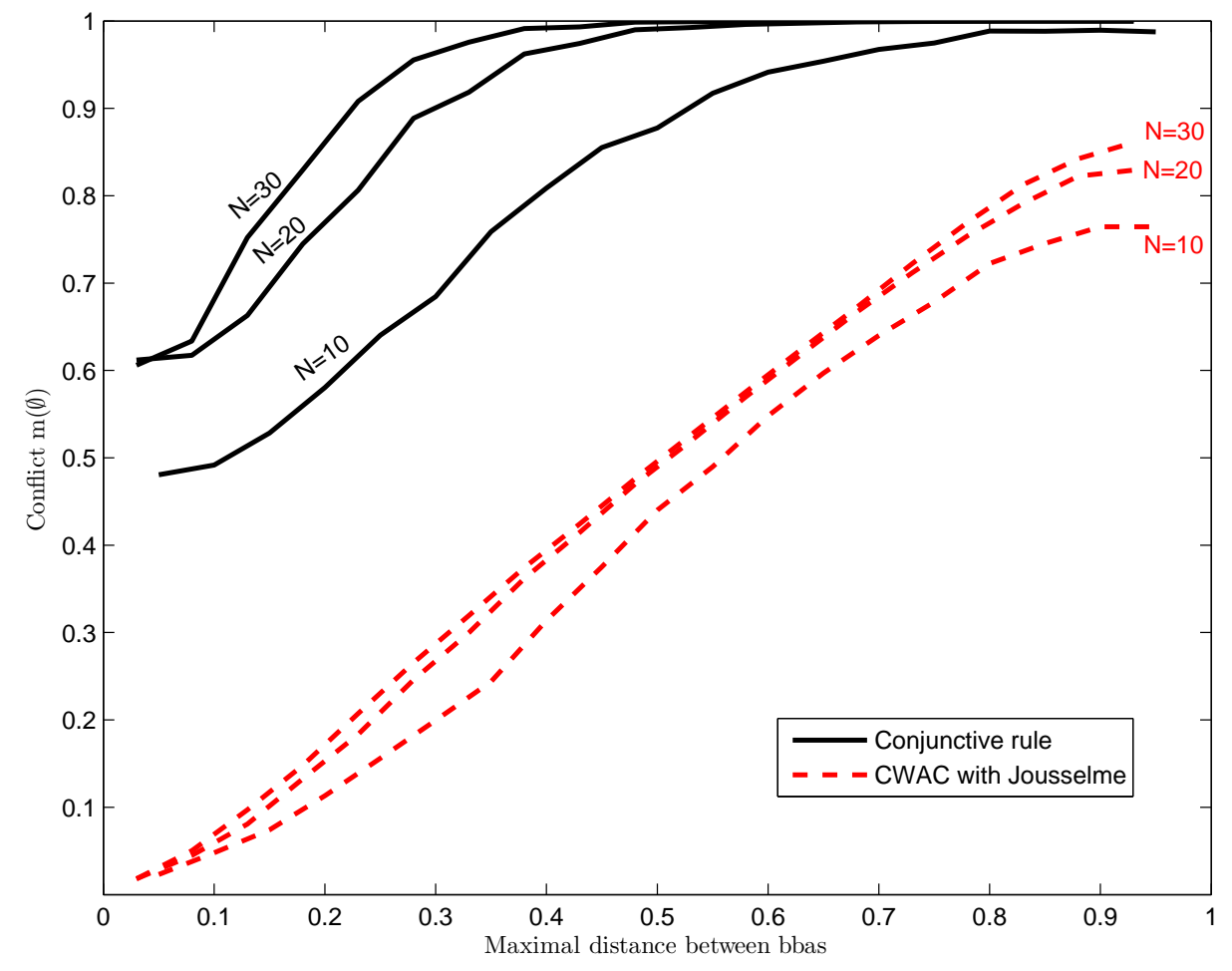

Figure 1: Behavior of conflict when the distance between bbas varies. 
both sets, bbas are randomly generated so as to obtain maximal distances according to 3 categories:

- $D^{\prime}$ category: where the maximal distance between bbas of the two sets is included in $[0 ; 0.3]$

- $D^{\prime \prime}$ category: where the maximal distance between bbas of the two sets is included in $] 0.3 ; 0.6]$

- $D^{\prime \prime \prime}$ category: where the maximal distance between bbas of the two sets is included in ]0.6; 0.9]

The generation of bbas is realized in the following way. We generate randomly, according to a normal law, the focal elements of the bba belonging to the set $S_{1}$. Then, we generate, according to a normal law, the degree of belief to be assigned to each of these focal elements. The same process will be repeated for bbas belonging to the set $S_{2}$ by verifying the constraint imposed on the maximal distance. Once these bbas were obtained, we duplicate them in each of the sets according to the wished proportion. For that purpose, we note $r$ the proportion of the set $S_{1}$ which expresses itself in the following way:

$$
r=\frac{\left|S_{1}\right|}{N}
$$

Where $N=\left|S_{1}\right|+\left|S_{2}\right|$ and $|$.$| represent the cardinal of the considered set.$

In this example, we consider $N=20$ and $|\Omega|=3$. This experiment is 100 times realized and then the results are averaged and represented in Figure 2 with Jousselme's distance.

On these figures, when the proportion is equal to 0 or to 1 , the involved bbas in the fusion process belong all to the same set. In that case, the conjunctive combination gives nevertheless an important conflicting mass ${ }^{5}$.

The evolution of $r$ only modifies very slightly this value especially in the case of a low distance. In the case of our CWAC operator, when all the bbas arise from the same set $(r=0$ or $r=1)$, the conflicting mass is null.

As soon as a singular source is involved in the fusion process, our rule allows to reach a maximal value which will remain unchanged. This maximum stays in reasonable proportions when the maximal distance is included

\footnotetext{
${ }^{5}$ This value is lesser when the distance is high because in that case the generated bbas are more categorical.
} 


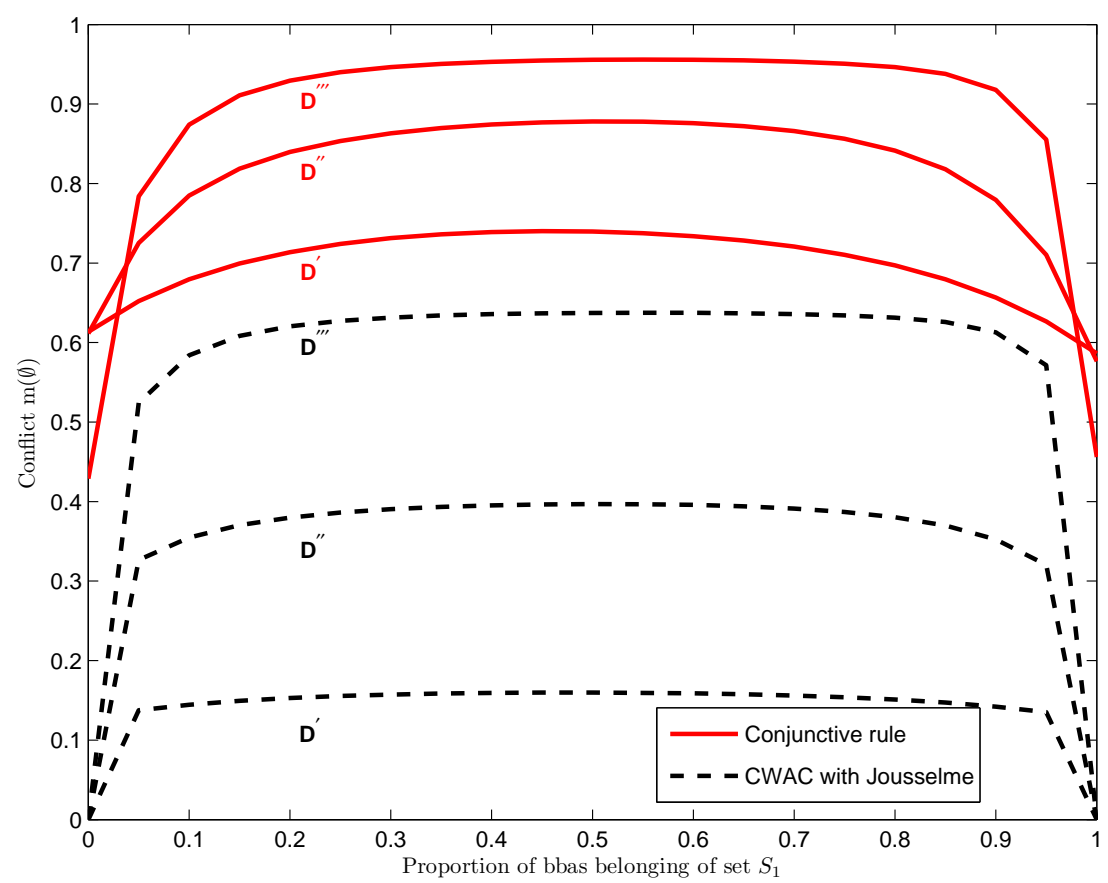

Figure 2: Behavior of conflict when the proportion of conflictual bbas varies. The CWAC operator is obtained with Jousselme's distance.

between [0;0.3], that is when bbas of both sets are considered as relatively similar. This remark is not verified in case of the conjunctive combination.

\section{Conclusion}

In this paper, we have proposed an approach with adapted conflict preserving the initial role of the conflict which is an alarm signal for the combination of belief functions. CWAC presents an adaptive weighting between Dempster's and conjunctive rules based on Jousselme et al.'s distance. Experimentations have shown that through CWAC, the absorbing power of the conflict is reduced compared with the conjunctive rule of combination. As future works, we intend to deal with the interpretation of the conflict when sources are dependent. Besides, the dual of CWAC will be proposed in the disjunctive behavior where the frame of discernment $\Omega$ is the absorbing element. 


\section{References}

[1] M. Bauer. Approximations algorithm and decision making in the Dempster-Shafer theory of evidence - an empirical study. International Journal of Approximate Reasoning, 17(2-3):217-237, 1997.

[2] F. Cuzzolin. Two new Bayesian approximation of belief functions based on convex geometry. IEEE Transactions on Systems, Man and Cybernetics - Part B, 37(4):993-1008, 2007.

[3] F. Cuzzolin. Geometric conditioning of belief functions. In Proceedings of 1st Workshop on the Theory of Belief Functions, 2010.

[4] M. Daniel. Associativity in combination of belief functions; a derivation of minC combination. Soft computing A Fusion of foundation, Methodologies and Application, 7(5):288-296, 2003.

[5] M. Daniel. Conflicts within and between belief functions. In Proceedings of 13th International Conference on Information Processing and Management of Uncertainty (IPMU'10), pages 696-705. Springer-Verlag, 2010.

[6] M. Daniel. Non-conflicting and conflicting parts of belief functions. In Proceedings of 7th International Symposium on Imprecise Probability: Theories and Applications (ISIPTA'11), pages 149-158, 2011.

[7] F. Delmotte, L. Dubois, A.M. Desodt, and P. Borne. Using trust in uncertainty theories. Information and Systems Engineering, 1:303-314, 1995.

[8] A.P. Dempster. Upper and lower probabilities induced by a multivalued mapping. Annals of Mathematical Statistics, 38:325-339, 1967.

[9] T. Denoeux. The cautious rule of combination for belief functions and some extensions. In Proceedings of International Conference on Information Fusion (FUSION'06), 2006.

[10] T. Denoeux. Conjunctive and disjunctive combination of belief functions induced by non distinct bodies of evidence. Artificial Intelligence, 172(23):234-264, 2008. 
[11] S. Destercke and T. Burger. Toward an axiomatic definition of conflict between belief functions. IEEE Transactions on Systems, Man and Cybernetics - Part B, 13(2):585-596, 2013.

[12] D. Dubois and H. Prade. A set theoretic view of belief functions: Logical operation and approximations by sets. International Journal of General Systems, 12(3):193-226, 1986.

[13] D. Dubois and H. Prade. Representation and combination of uncertainty with belief functions and possibility measures. Computational Intelligence, 4:244-264, 1988.

[14] D. Dubois and H. Prade. Reliability Data Collection And Analysis, chapter On the combination of evidence in various mathematical frameworks, pages 213-241. J. Flamm and T. Luisi, Brussels, 1992.

[15] E. Elouedi, E. Lefevre, and D. Mercier. Discountings of a belief function using a confusion matrix. In 22th IEEE International Conference on Tools with Artificial Intelligence (ICTAI'2010), volume 1, pages 287294, 2010.

[16] Z. Elouedi, K. Mellouli, and P. Smets. Assessing sensor reliability for multisensor data fusion with the Transferable Belief Model. IEEE Transactions on Systems, Man and Cybernetics, Part B, 34:782-787, 2004.

[17] D. Fixsen and R.P.S. Mahler. The modified Dempster-Shafer approach to classification. IEEE Transactions on Systems, Man and Cybernetics Part A, 27:96-104, 1997.

[18] M.C. Florea, J. Dezert, P. Valin, F. Smarandache, and A-L. Jousselme. Adaptative combination rule and proportional conflict redistribution rule for information fusion. In Proceedings of COGnitive Systems with Interactive Sensors (COGIS'06), 2006.

[19] M.C. Florea, A-L. Jousselme, E. Boisé, and D. Grenier. Robust combination rules for evidence theory. Information Fusion, 10(2):183-197, 2009.

[20] T. George and N. R. Pal. Quantification of conflict in dempster-shafer framework: A new approach. International Journal of General Systems, 24(4):407-423, 1996 
[21] A. Josang. The consensus operator for combining beliefs. Artificial Intelligence, 141(1-2):157-170, 2002.

[22] A. Josang, J. Diaz, and M. Rifqi. Cumulative and averaging fusion of beliefs. Information Fusion, 11(2):192-200, 2010.

[23] A-L. Jousselme, D. Grenier, and E. Boissé. A new distance between two bodies of evidence. Information Fusion, 2:91-101, 2001.

[24] A-L. Jousselme and P. Maupin. On some properties of distance in evidence theory. In Proceedings of 1st Workshop on the Theory of Belief Functions, 2010.

[25] A-L Jousselme and P. Maupin. Distances in evidence theory: Comprehensive survey and generalizations. International Journal of Approximate Reasoning, 53(2):118-145, 2012.

[26] J. Klein and O. Colot. Automatic discounting rate computation using a dissent criterion. In Proceedings of 1st Workshop on the Theory of the Belief Functions, 2010.

[27] J. Klein and O. Colot. Singular sources mining using evidential conflict analysis. International Journal of Approximate Reasoning, 52(9):14331451, 2011.

[28] E. Lefevre, O. Colot, and P. Vannoorenberghe. Belief function combination and conflict management. Information Fusion, 3:149-162, 2002.

[29] E. Lefevre, Z. Elouedi, and D. Mercier. Towards an alarm for opposition conflict in a conjunctive combination of belief functions. In 11th European Conference on Symbolic and Quantitative Approaches to Reasoning with Uncertainty (ECSQARU'11), pages 314-325. Springer-Verlag, 2011.

[30] W. Liu. Analyzing the degree of conflict among belief functions. Artificial Intelligence, 170(11):909-924, 2006.

[31] Z. G. Liu, J. Dezert, Q. Pan, and G. Mercier. Combination of sources of evidence with different discounting factors based on a new dissimilarity measure. Decision Support Systems, 52(1):133-141, 2011. 
[32] Z. G. Liu, Q. Pan, Y-M. Cheng, and J. Dezert. Sequential adaptative combination of unreliable sources of evidence. In Proceedings of 1 st Workshop on the Theory of Belief Functions, 2010.

[33] A. Martin, A.L. Jousselme, and C. Osswald. Conflict measure for the discounting operation on belief functions. In Proceedings of International Conference on Information Fusion (FUSION'08), pages 10031010, 2008.

[34] A. Martin and C. Osswald. Human experts fusion for image classification. Information Security: An International Journal, Special issue on fusing uncertain, imprecise and paradoxist information (DSmT), 20:122143, 2006.

[35] A. Martin and C. Osswald. Toward a combination rule to deal with partial conflict and specificity in belief functions theory. In Proceedings of International Conference On Information Fusion (Fusion'07), 2007.

[36] D. Mercier, G. Cron, T. Denoeux, and M.-H. Masson. Decision fusion for postal address recognition using belief functions. Expert Systems with Applications, 36(3, part 1):5643-5653, 2009.

[37] C.K. Murphy. Combining belief functions with evidence conflicts. Decision Support Systems, 29(1):1-9, 2000.

[38] C. Osswald and A. Martin. Understanding the large family of DempsterShafer theory's fusion operators - a decision-based measure. In Proceedings of International Conference On Information Fusion (FUSION'06), 2006.

[39] B. Ristic and P. Smets. The TBM global distance measure for the association of uncertain combat ID declaration. Information Fusion, 7:276-284, 2006.

[40] J. Schubert. Specifying nonspecific evidence. International Journal of Intelligence Systems, 11:525-563, 2006.

[41] J. Schubert. Conflict management in Dempster-Shafer theory using the degree of falsity. International Journal of Approximate Reasoning, 52(3):449-460, 2011. 
[42] J. Schubert. The internal conflict of a belief function. In 2rd International Conference on Belief Functions, pages 169-177. Springer Verlag, 2012.

[43] G. Shafer. A Mathematical Theory of Evidence. Princeton University Press, Princeton, NJ, 1976.

[44] P. Smets. The combination of evidence in the Transferable Belief Model. IEEE Transactions on Pattern Analysis and Machine Intelligence, 12(5):447-458, 1990.

[45] P. Smets. Belief functions: The disjunctive rule of combination and the generalized Bayesian theorem. International Journal of Approximate Reasoning, 9:1-35, 1993.

[46] P. Smets. The canonical decomposition of a weighted belief. In Inter. Joint Conf. On Artificial Intelligence, pages 1896-1901, San Matco, CA, 1995. Morgan Kaufman.

[47] P. Smets. Analyzing the combination of conflicting belief functions. Information Fusion, 8(4):387-412, 2007.

[48] P. Smets and R. Kennes. The Transferable Belief Model. Artificial Intelligence, 66:191-234, 1994.

[49] B. Tessem. Approximations for efficient computation in the theory of evidence. Artificial Intelligence, 61(2):315-329, 1993.

[50] A. Veremme, E. Lefevre, G. Morvan, D. Dupont, and D. Jolly. Evidential calibration process of multi-agent based system: An application to forensic entomology. Expert Systems with Applications, 39(3):2361-2374, 2012.

[51] R. Yager. On the Dempster-Shafer framework and new combination rules. Information Sciences, 41:93-138, 1987.

[52] K. Yamada. A new combination of evidence based on compromise. Fuzzy sets and systems, 159(13):1689-1708, 2008.

[53] D. Yong, S. Wenkang, Z. Zhenfu, and L. Qi. Combining belief functions based on distance of evidence. Decision Support Systems, 38(3):489-493, 2004 . 
[54] L. Zhang. Representation, independence and combination of evidence in the Dempster-Shafer theory. Book title Advances in the Dempster-Shafer Theory of Evidence, pages 51-69, 1994.

[55] L.M. Zouhal and T. Denoeux. An evidence-theoretic k-NN rule with parameter optimization. IEEE Transactions on Systems, Man and $C y$ bernetics - Part C, 28(2):263-271, 1998. 